\title{
ARTIGO DE ATUALIZAÇÃo Estigma social sobre o uso de álcool
}

\author{
Social stigma about alcohol use \\ Telmo Mota Ronzani', Erikson Felipe Furtado²
}

\section{Palavras-chave}

Estigmatização,

estereotipagem, transtornos

relacionados ao uso de

substâncias.

\section{Keywords}

Stigmatization, stereotyping, substancerelated disorders.

\section{RESUMO}

O uso de álcool e outras drogas é um importante problema de saúde pública em todo o mundo, com relativa complexidade no entendimento do tema e nas ações de prevenção e tratamento. O objetivo do presente estudo é apresentar uma revisão bibliográfica sobre estigma social e uso de álcool. Estudos que investigam o estigma social associado ao usuário de álcool e de outras drogas sugerem que tal associação apresenta um importante impacto tanto para a definição de políticas públicas quanto para ações de prevenção e tratamento. A moralização e os estereótipos associados aos usuários podem influenciar direta ou indiretamente nos resultados e na qualidade da prevenção e tratamento do uso. Dessa forma, estudos e ações que visem diminuir o impacto do estigma na qualidade de assistência de usuários tornam-se relevantes.

\begin{abstract}
The use of alcohol and other drugs is an important public health problem worldwide with certain complexity in understanding about the subject and in prevention and treatment actions. The aim of this paper is to present a bibliographic revision about social stigma and alcohol use. Studies that investigate the social stigma related with alcohol and other drugs users suggest that such association presents an important impact both in definition of public policy and in prevention and treatment actions. Moralization and stereotype associated to users can influence direct or indirectly the quality and outcomes of treatment and prevention of drug use. Nevertheless, studies and actions that aim to decrease the impact of stigma in quality of users assistance are relevant.
\end{abstract}

\section{INTRODUÇÃO}

O uso de álcool é um comportamento presente em diversas culturas, sociedades e na história humana, havendo, de forma geral, um conteúdo ritualístico em tal comportamento. Porém, com a modernização e a mudança da organização social em algumas culturas e com as transformações econômicas e sociais, houve uma mudança na forma como os indivíduos e grupos passaram a utilizar o álcool. Esse comportamento tem sido associado principalmente ao contex- to recreativo ou à busca imediata de prazer, com padrões de uso em grandes quantidades numa única ocasião ou ao longo do tempo, característica que coloca o consumo atualmente como um dos principais problemas de saúde pública no mundo ${ }^{1-3}$.

É cada vez mais evidente a complexidade envolvida no consumo de álcool, sendo fundamentais abordagem e entendimento ampliados sobre o tema ${ }^{4}$. Além disso, dentro da atual realidade epidemiológica do Brasil e do mundo, com o aumento constatado da incidência de doenças crônicas, os

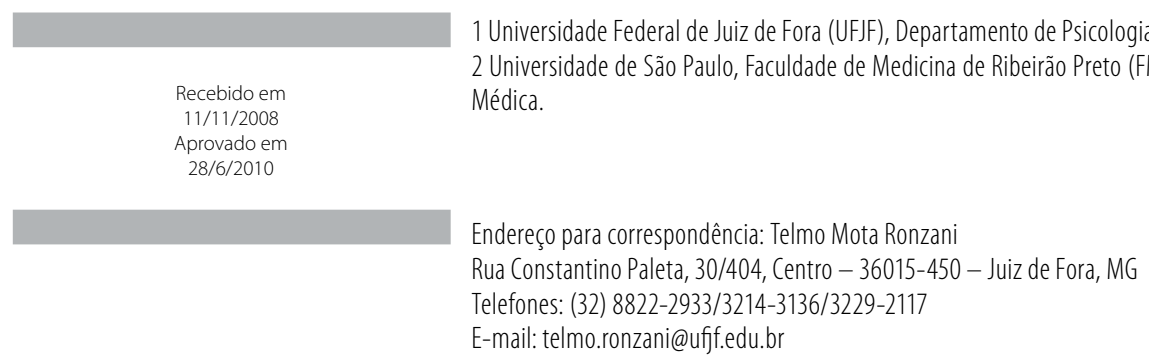


aspectos comportamentais envolvidos no tratamento e na prevenção da maioria das doenças apresentam importância cada vez maior. As atitudes que a população ou profissionais apresentam sobre determinada situação de saúde são fundamentais para a qualidade do atendimento, adesão ao tratamento e atividades de prevenção ${ }^{5,6}$.

Portanto, muito além dos aspectos físicos da ingestão ou problemas orgânicos associados, o uso de álcool apresenta resultados diferenciados de acordo com os aspectos sociais ${ }^{7}$. Dentre tais aspectos, a moralização ou estigmatização do uso pode influenciar de forma importante tanto nas consequências do uso como no resultado do tratamento ou acesso a este ${ }^{6}$.

Muitas condições de saúde, entre elas a saúde mental e a dependência de álcool e outras substâncias, são estigmatizadas pela população ou pelos profissionais de saúde $e^{5,6,8}$. A estigmatização ocorre quando pessoas atribuem rótulos e estereótipos negativos a determinados comportamentos ${ }^{9}$. Tal condição influencia direta ou indiretamente a condição de saúde da pessoa estigmatizada, levando a diversas consequências, inclusive ao agravamento da situação ${ }^{5,10}$. Em pesquisa realizada por Fortney et al. ${ }^{6}$, observou-se que quanto maior o sentimento de estigmatização percebido pelos usuários de álcool por parte dos profissionais de saúde, menores a adesão e a qualidade do atendimento a tais usuários.

Especificamente nas perspectivas assistenciais no campo da prevenção ao uso de risco de álcool, algumas estratégias de triagem e intervenções breves para o uso abusivo de substâncias estão sendo avaliadas para populações específicas, principalmente no contexto da Atenção Primária à Saúde (APS), em todo o mundo e mais recentemente no Brasil ${ }^{11-20}$.

Por tal razão, a Organização Mundial da Saúde (OMS) vem desenvolvendo há alguns anos estudos multicêntricos em diversos países, com o objetivo de avaliar a implementação de rotinas de triagem e intervenções breves para o uso de álcool em serviços de APS. A ênfase de tais estudos tem sido na avaliação do impacto do treinamento de profissionais de saúde e da supervisão continuada na mudança de atitudes dos profissionais e na incorporação das práticas de prevenção ao uso de risco na rotina dos serviços 3,11,20.

A partir de estudos anteriores, que têm avaliado a implementação de estratégias de prevenção ao uso de risco de álcool, uma das principais barreiras para a implementação de tais ações no Brasil tem sido as atitudes negativas que os profissionais de saúde têm sobre o uso e o usuário de álcool 1,11,20. Sendo assim, estudos que avaliem quais são as atitudes dos profissionais e até que ponto elas influenciam nas barreiras para efetivar cuidados adequados aos usuários são fundamentais. É importante ressaltar que tais atitudes encontradas nos estudos anteriores apresentam um substrato contextual importante, pois as atitudes negativas sobre determinado comportamento social seriam formadas, segundo a Teoria da Aprendizagem Social ${ }^{21}$, pelas normas sociais, que variam de acordo com o grupo em que os indivíduos estão inseridos e com o contexto sócio-histórico e cultural em que se situam ${ }^{22}$.

Um dos contextos de saúde estratégicos para se estudar e mudar o processo de estigmatização dos usuários de álcool é o da APS. A APS é um nível de atenção importante, pois os profissionais têm contato com um grande número de pessoas, que não são exatamente pacientes de nível especializado, sendo um nível de atenção estratégico para as práticas preventivas e, consequentemente, para um trabalho também preventivo sobre as percepções e estigmatização dos usuários de álcool e outras substâncias, facilitando o acesso de uma abordagem adequada dos usuários iniciais 4,13,18,19,23-25.

O objetivo do presente artigo é realizar uma revisão teórica sobre o processo de estigmatização sobre o uso e os usuários de álcool no contexto da saúde coletiva.

\section{O PROCESSO DE ESTIGMATIZAÇÃO DE PROBLEMAS DE SAÚDE}

O estudo sobre o estigma social tem como marco teórico fundamental a obra clássica de Goffman ${ }^{26}$, que descreve o estigma como um sinal ou uma marca que designa o portador como "deteriorado" e, portanto, menos valorizado do que as pessoas "normais".

Poderíamos, a partir desse conceito inicial, definir o estigma social como uma marca física ou social de conotação negativa ou que leva o portador dessa "marca" a ser marginalizado ou excluído de algumas situações sociais ${ }^{27}$, apresentando forte impacto no valor atribuído a uma determinada identidade social. Como característica fundamental, a estigmatização tem como base central sua derivação cultural ou situacional e envolve os seguintes componentes, de acordo com Dovidio et al.28: 1) o reconhecimento da diferença com base em alguma característica distinguível, ou "marca"; 2) a consequente desvalorização da pessoa estigmatizada. Em estudos mais recentes, tem-se defendido a natureza dinâmica, relacional e contextual do estigma.

O estigma social pode causar forte impacto à vida para a pessoa estigmatizada, pois envolve aspectos amplos à vida dos sujeitos, assim como a formação e a transformação da identidade social desvalorizada num dado contexto social. Indivíduos estigmatizados são tidos como "imperfeitos", "comprometidos" e de alguma forma teriam um atributo em algumas situações mais extremas desumanizadas ${ }^{10,28,29}$. Por causa dessas características, a estigmatização apresenta profundas consequências negativas, e mesmo patológicas para a personalidade dos estigmatizados, resultando em estratégias e enfrentamento ou fuga de algumas situações que podem se tornar prejudiciais aos indivíduos ${ }^{30-32}$. Levando em consideração o forte substrato sociocultural do estigma, o 
contexto tem, portanto, um forte papel em relação ao nível de consequências para o indivíduo estigmatizado ${ }^{31}$.

Tais consequências envolvem também um distanciamento social que pode representar consequência direta ou indireta à saúde e ao bem-estar de quem é estigmatizado. Essas consequências, de forma geral, podem ser indiretas, como a dificuldade de acesso a cuidados em saúde, educação, emprego, moradia e outros ${ }^{5,30}$.

\section{Como surge e é mantido o estigma}

Como já afirmado anteriormente, o processo de estigmatização possui um substrato sociocultural e é parte de atitudes a respeito do objeto estigmatizado. Por sua vez, as atitudes são formadas por uma organização duradoura de crenças e cognições, afetos positivos ou negativos diante de uma situação social. Nesse modelo de concepção de atitude, portanto, deve-se entender que os três componentes estão presentes de forma articulada e interdependente e relacionados, ainda, ao contexto social ${ }^{33}$. Nessa concepção, de forma geral, a estigmatização é mantida e/ou formada por meio do componente cognitivo das atitudes (crenças) que predispõem a determinados comportamentos ou sentimentos sobre a situação estigmatizada $21,28,34$.

Pode-se observar, então, que as crenças são formadas e, principalmente, mantidas pela percepção, que apresenta forte aspecto social e cria um direcionamento atencional para determinados aspectos da percepção. Dessa maneira, as crenças atuam entre a percepção de um determinado objeto (grupo) e a representação do objeto em si, havendo uma situação social que influencia o significado e a reação a respeito dessa percepção, levando o indivíduo a ter uma tendência classificatória ou de rotulação sobre outras pessoas ${ }^{10,21}$.

Deve-se entender que as crenças podem ser alteradas por algum dos aspectos da atitude descritos acima ou ainda pela mudança de algum aspecto social. Dependendo da centralidade psicológica das crenças e de aspectos contextuais, elas seriam mais fáceis ou difíceis de serem alteradas ${ }^{35}$. Determinadas crenças ainda podem se tornar sociais, a partir do compartilhamento entre grupos e sociedades, podendo haver uma generalização que terá certa durabilidade, formando alguns estereótipos. Tais estereótipos ou "estigmas sociais" influenciam a relação entre a população e as pessoas que carregam tal estigma .

Esse mecanismo de generalização, presente no processo de estigmatização e estereotipização, leva a reações automáticas, por questões de economia psíquica, quando a mera presença de uma característica facilmente discernível seria suficiente para desencadear um processo automático de estereotipia. Esse processo é denominado por Lima e Vala ${ }^{9}$ de "Monstro da Automaticidade Cognitiva". Um aspecto importante da automaticidade é que os estereótipos são formados a partir de determinados moderadores sociais, sendo, portanto, importante entender o contexto no qual determina- dos estereótipos são formados, para conhecer o processo de estigmatização de alguns grupos ${ }^{34}$.

Nesse processo complexo e relacional, pode-se entender que as atitudes representariam um resumo de avaliação de um objeto psicológico capturado em dimensões de atributos como bom-ruim, danoso-benéfico, prazeroso-desprazeroso, desejável-indesejável. A partir de tais processos, indivíduos tendem a manter, com certo grau de maleabilidade, suas tendências crônicas para se agarrar a padrões de resposta. Dessa maneira, pode-se entender como as atitudes e a estigmatização são formadas, mantidas e mudadas ${ }^{34}$.

Na sociedade moderna, um dos principais meios de formação, manutenção e mudança de crenças e atitudes é a opinião pública ${ }^{36}$. Segundo Ramos ${ }^{36}$, ao longo da história, a opinião pública deixa de ser difundida pelo encontro direto entre pessoas ou grupos, e a mídia de massa se torna o mediador e divulgador de ideias sobre determinados eventos. Tal mudança se deve, principalmente, à urbanização e à modernização das sociedades. A imprensa, portanto, veio caracterizar-se definitivamente como principal agente da opinião, tendo seu alcance e seu poder de influência aumentados.

Além de sua importância enquanto meio de propagação de ideias, a mídia se torna uma fonte de identificação fundamental para os sujeitos ou grupos como forma de localização social de uma opinião pessoal. É interessante ressaltar que a mídia, além de ser uma fonte de formação de crenças e atitudes, também pode representar um contexto específico, podendo, portanto, refletir o pensamento coletivo em um determinado momento ou influenciar tal pensamento ${ }^{36}$. Portanto, os meios de comunicação de massa tornam-se um importante veículo de fortalecimento e difusão de ideologias específicas com grande poder de alcance ${ }^{37}$.

\section{Estigma e outros conceitos relacionados}

Como se pode observar, a conceituação e o entendimento do estigma social estão relacionados a diversas outras instâncias conceituais, uma vez que este não se constitui em um conceito isolado na literatura atual ${ }^{31,32}$.

Na presente seção, serão apresentados os principais conceitos relacionados ao de estigma social. O primeiro conceito apresentado é o de estereótipos. Pode-se dizer que o estereótipo é um mecanismo cognitivo básico de supergeneralização e prototipização de indivíduos ou grupos, como forma de economia psíquica perante diversas informações e estímulos sociais ${ }^{9,38,39}$. Os pensamentos estereotipados tipicamente servem a múltiplas propostas que refletem uma variedade de processos cognitivos e motivacionais, podendo simplificar o processamento de informação, e podem emergir como respostas a situações ambientais ou ainda para justificar o status quo de uma situação dada. Enfim, os estereótipos emergem em vários contextos para ter uma função demandada por esse contexto ${ }^{38}$. Os conceitos de estereótipos e estigma estão extremamente associados, porém exis- 
tem algumas diferenças básicas. A principal diferenciação é que os estereótipos podem existir sem estigmas e alguns estereótipos podem ser positivos, enquanto a estigmatização é sempre negativa. Além disso, nem sempre um estereótipo negativo se constitui em um estigma, que envolve aspectos mais amplos. Porém, pode-se observar mais semelhanças e relações entre os conceitos do que diferenças. Ambas envolvem o reconhecimento de marcas que o grupo identifica como uma categoria de pertencimento. Além disso, a estereotipização pode ter um papel central no desenvolvimento, manutenção e perpetuação da estigmatização. Estereótipos formam um conjunto de crenças que guiam o processamento da informação e atribuições, podendo produzir profecias autorrealizadoras e servir como fonte de justificativa de um determinado estigma ${ }^{31}$.

Outros conceitos relacionados ao estigma são o de marginalidade e desvio. O primeiro estaria relacionado ao pertencimento a um grupo social estatisticamente incomum sobre certo atributo. Já o desvio seria entendido como um comportamento ou condição que envolve um indesejável desvio da normalidade em relação a um padrão específico. Uma pessoa estigmatizada pode ser percebida como desviante ou marginalizada ante uma situação específica. Por outro lado, pode haver pessoas desviantes ou marginalizadas por causa de características sociais tidas como positivas (como riqueza extrema, por exemplo), mas que não são necessariamente estigmatizadas por isso. Além disso, mesmo que a maioria das condições desviantes ou marginalizadas seja vista como negativa, é preciso haver uma condição mais ampla e que envolva o comprometimento da identidade do portador como eminentemente ruim por tal característica. Enfim, estigma envolve condições de marginalização e desvio, mas prescinde de atribuições mais gerais sobre o caráter e a identidade do estigmatizado 28 .

Por último, o estigma também está relacionado ao preconceito, e a pessoa estigmatizada é quase sempre alvo de preconceito. Preconceito é amplamente definido como uma atitude negativa, relativamente inflexível, que predispõe a pessoa que apresenta o preconceito a determinados comportamentos ou profecias autorrealizadoras. Embora o estigma esteja amplamente relacionado com o preconceito, o primeiro conceito apresenta abrangência e amplitude conceituais maiores ${ }^{28}$.

\section{O USO DE ÁLCOOL COMO PROBLEMA DE SAÚDE PÚBLICA}

Em vários estudos realizados em alguns países, verifica-se em parcela significativa da população a ocorrência de padrões de consumo de álcool com elevado grau de risco para diversos problemas de saúde, psicológicos e sociais 2,13,26,40. Tais dados refletem no aumento de 1,5\% da taxa de morbi- mortalidade atribuível ao uso de álcool em 1990 para 3,2\% em 2000, havendo um aumento de mais que o dobro no valor encontrado no período de dez anos, indicando, portanto, uma tendência preocupante em termos de saúde pública.

O álcool é responsável por 3,2\% das mortes e 4,0\% de AVAls (ou DALYs - anos de vida ajustados por incapacidade) ${ }^{40}$. Na América Latina, o álcool se torna um problema mais evidente, sendo a principal causa de carga global de doenças em população acima de 15 anos $^{41}$. Estima-se um consumo médio percapita de 6,7 litros de álcool puro por ano, com tendência de aumento de consumo ao longo dos anos ${ }^{41}$.

No Brasil, estima-se que 12,3\% da população geral sejam dependentes de álcool, com prevalência crescente ao longo dos anos, segundo estudo do Centro Brasileiro de Informações sobre Drogas Psicotrópicas (Cebrid) ${ }^{42}$. Além da dependência em si, algumas informações epidemiológicas sobre o uso de álcool sugerem que esse comportamento não se limita ao problema da dependência em si, mas a outros problemas como violência, problemas crônicos e agudos de saúde associados, acidente de trânsito etc. ${ }^{43,44}$, havendo início de uso cada vez mais cedo entre os jovens ${ }^{45}$ e consumo cada vez maior entre as mulheres ${ }^{44}$.

Além disso, o uso de risco ou abusivo de álcool pode ser prejudicial aos indivíduos e à população geral, por causa dos problemas relacionados a tal comportamento ${ }^{13,42}$. Mais de 60 patologias crônicas e agudas, além de outros problemas sociais e psicológicos, estão associadas ao uso de álcool ${ }^{3,18,25}$. Por essa razão, um entendimento mais amplo e cuidadoso sobre o uso de álcool deve ser contemplado, investindo-se em políticas públicas adequadas e voltadas para ações preventivas aos vários problemas associados ao uso como problemas crônicos ou agudos, violência, acidentes automobilísticos, comportamento sexual de risco, tentativas de suicídio, problemas familiares, laborais e no rendimento acadêmico ${ }^{2,46}$.

Outro dado considerável é que no Brasil, em média, são consumidos anualmente 6 litros de álcool per capita. Além da quantidade ingerida, o padrão de consumo pode levar a diversos problemas de saúde devidos ao uso de altas doses numa mesma ocasião (padrão Binge) ${ }^{47}$. Em estudo nacional recente sobre o padrão de uso de álcool no Brasil, 38\% dos homens e $17 \%$ das mulheres fizeram um consumo de cinco doses ou mais numa única ocasião no último ano, sendo esse uso mais pesado entre os jovens, com diferenças menores entre os gêneros. Tais dados demonstram que o consumo de álcool entre brasileiros os coloca em situação de risco para diversos problemas sociais e de saúde ${ }^{45}$.

Dados semelhantes foram encontrados entre usuários de serviços de APS. Em estudo realizado com tal população, $22,2 \%$ dos pacientes faziam uso de risco de álcool, com uso frequente (todos ou quase todos os dias) de 19,3\% de homens e 7,2\% de mulheres. Além disso, chama atenção que $34,7 \%$ dos pacientes de APS fizeram uso de mais de cinco doses numa única ocasião ${ }^{47}$. Considerando que essa popu- 
lação tipicamente apresenta faixa etária mais avançada, as consequências de um padrão de uso de bebida mais pesado podem acarretar problemas mais graves.

Tendo em vista os dados epidemiológicos apresentados e o impacto que o uso de álcool pode causar para a vida das pessoas e populações, ações concretas de prevenção ao uso de risco são necessárias e um cuidado adequado deve ser oferecido nos diversos conceitos. Sabe-se, porém, que muitas vezes o acesso aos serviços e aos cuidados é dificultado por causa do estigma atribuído ao uso nocivo de álcool, fato que pode agravar a situação do usuário, uma vez que ele procura ajuda pensando que receberá um julgamento moralizado por parte dos profissionais de saúde 6 . Esse processo pode ocorrer de algumas maneiras, como definido na próxima sessão do artigo.

\section{ESTIGMATIZAÇÃO DO USO/USUÁRIO DE ÁLCOOL}

Pode-se observar que a estigmatização traz um impacto importante para a vida de pessoas e grupos e que tais impactos normalmente residem não nos resultados físicos de uma marca em si, mas em suas consequências sociais e psicológi$\operatorname{cas}^{28}$. No processo de formação do estigma social, as características negativas atribuídas são reconhecidas não somente pelo grupo externo, mas também internalizado pelas pessoas portadoras desse estigma, influenciando diretamente as crenças e sentimentos das pessoas em relação às características atribuídas como negativas ${ }^{28,47}$. Nesse sentido, muitas vezes, em situações de saúde, por exemplo, a condição de estigmatização pode se tornar muito mais danosa do que propriamente a doença em si.

No campo da saúde coletiva, o processo de estigmatização é evidente em algumas áreas específicas. Tal processo começa já na definição do diagnóstico. A despeito da importância do diagnóstico como uma ferramenta importante para a definição de um tratamento mais adequado, muitas vezes, a conotação que se dá para determinados problemas é muito mais vinculada a uma questão moral ${ }^{8}$. Muitos profissionais lidam com alguns problemas de saúde de forma compartiIhada com a população geral, na qual se tende a classificar as pessoas em parâmetros de normalidade ou não, definindo alguns comportamentos desviantes na sociedade ${ }^{7,29}$.

Dentre os vários aspectos envolvidos no processo de estigmatização, a generalização estereotipada e a atribuição moral de determinados comportamentos merecem destaque $^{30}$. No campo da saúde, a conotação moral envolvida em alguns processos de diagnóstico se torna um início do processo de estigmatização de algumas condições. O julgamento moral envolve a concepção de algo como "problema" indesejável de se lidar, sendo considerado negativo. Nesse processo, tanto o problema em si quanto o portador desse problema são generalizados como um problema indesejável ${ }^{30}$, influenciando diretamente no planejamento, no acesso e na responsabilidade sobre o tratamento por parte do profissional de saúde ${ }^{5,45}$.

Dentre as diversas condições de saúde comumente estigmatizadas pela sociedade em geral e pelos profissionais de saúde, a saúde mental é reconhecidamente uma das áreas em que o estigma social é mais presente ${ }^{31,48}$, influenciando direta ou indiretamente no tratamento e na qualidade de vida das pessoas com tal condição de saúde. Estudos demonstram que pessoas com transtornos mentais são vistas como mais responsáveis por sua condição do que aquelas com doenças como câncer, problemas cardíacos ou outras doenças $^{49}$. Esses dados refletem na forma como os profissionais de saúde ou políticas públicas lidam com o problema, uma vez que a atribuição de responsabilidade sobre determinado problema de saúde pode ser relacionada com a prontidão para a ajuda oferecida pelos profissionais e pela estigmatização e moralização da condição apresentada29,31,32,50.

O uso de álcool e outras drogas é uma das condições que mais apresentam uma conotação moralizante do mundo ${ }^{6,7,29,51}$, sendo considerado principalmente um problema individual, em que o diagnóstico e o tratamento muitas vezes exacerbam os aspectos morais do uso ${ }^{8,25,29}$. Durante muito tempo a classificação dos usuários de álcool se polarizou entre aqueles que faziam "uso social" e os que eram "dependentes". O estigma, carregado de conotações morais, associado ao "alcoólatra" ou "dependente de drogas", tem levado os pacientes, a sociedade e até mesmo os profissionais de saúde a resistirem em aceitar ou utilizar o diagnóstico de forma inadequada ${ }^{3,8}$.

A percepção ou danos associados ao problema do uso de álcool ou outras drogas também está associada a como a sociedade encara esse problema. Portanto, quando um paciente recebe o diagnóstico de alcoolista, além dos aspectos médicos ou psicológicos que caracteriza tal diagnóstico, a percepção social sobre esse problema influencia o peso pelo qual o paciente vai carregar tal diagnóstico 7,8 . Portanto, numa sociedade em que o alcoolismo ou uso de drogas apresenta uma forte conotação moralizante, o estigma social se torna um grande problema para o usuário. Muitas vezes, o profissional responsável pelo diagnóstico ou tratamento apresenta o pensamento de que o uso de álcool é um vício ou fraqueza de caráter ${ }^{25}$. A forma de abordagem dos usuários é influenciada por tal visão, e o tratamento é direcionado para a pessoa problemática, sendo necessário controlar o mau hábito e o comportamento desviante dessas pessoas ${ }^{29}$. Tal tipo de abordagem acaba por reforçar a perspectiva moralizante e a internalização de alguns estereótipos e estigmas atribuídos aos usuários de drogas.

Segundo Room ${ }^{25}$, existem duas vertentes de estudo sobre estigma do uso de álcool e outras drogas. A primeira é relacionada ao entendimento e ao estudo de como se forma 
o processo de estigmatização e de estratégias de mudança de percepção dos profissionais e dos próprios usuários sobre o uso de álcool, a fim de promover uma postura resiliente na recuperação do problema. Outra linha de pensamento estabelece que o reforçamento do estigma social é visto como benéfico no controle e na recuperação do usuário de álcool e outras drogas. De forma geral, segundo o autor, existe uma punição social não formalizada que procura passar a mensagem de que determinados comportamentos não são tolerados. Fortney et al. ${ }^{6}$, em seu estudo, observaram que a percepção de estigmatização de sua condição foi importante para a qualidade e o resultado do tratamento recebido, confirmando, portanto, a primeira linha de pensamento. Outros estudos demonstram também que o estigma é uma barreira para o tratamento ${ }^{5,25,29}$.

Como alternativa à concepção moralizante do usuário, procura-se estabelecer ações baseadas na perspectiva da saúde coletiva, em que o foco é dado ao uso de álcool e aos danos associados, e não aos indivíduos propriamente ditos, procurando traçar ações mais gerais, compreensivas e menos estigmatizantes, que também se articulem com ações direcionadas aos indivíduos, 8,18,25,29.

Uma das estratégias é o foco na mudança de posturas que levam à estigmatização do uso de álcool como forma de prevenir os danos associados no sentido de melhorar a eficácia e o acesso ao tratamento ${ }^{5,52}$. Essas ações são planejadas partindo-se do princípio de que a automaticidade e a estereotipização podem ser controladas ou modificadas por mediadores sociocognitivos ${ }^{9}$.

Para tanto, é importante considerar que as políticas assistenciais e a formação dos profissionais de saúde devem enfocar a mudança de atitudes negativas no sentido de evitar a estigmatização e a diminuição da consequente injustiça social que os portadores de sofrimento mental sofrem ${ }^{3,53}$.

\section{CONSIDERAÇÕES FINAIS}

A partir da presente revisão, pode-se chegar a algumas considerações importantes sobre o processo de estigmatização, que torna tal área de estudo importante não só para o campo de estudo no campo de álcool e outras drogas, mas também para a saúde coletiva.

Um primeiro aspecto a ser considerado é que a atribuição moral e a culpabilização/acusação do usuário por parte da população e em especial por parte dos profissionais de saúde podem criar barreiras na qualidade do cuidado em saúde, que levam à exclusão e ao calvário de alguns usuários dos serviços de saúde. Por essa razão, é preciso de estratégias que visem a mudança de atitudes, conhecimento e habilidades dos profissionais sobre os usuários de álcool e outras drogas. Existem alguns estudos demonstrando que, quando os profissionais possuem atitudes positivas em rela- ção ao usuário de álcool, eles se sentem tecnicamente mais preparados e motivados para realizar ações quanto aos cuidados com usuários de álcool e outras drogas ${ }^{6,48,53}$.

Outro aspecto a ser considerado é que as percepções sobre o uso ou usuários de álcool e outras drogas podem influenciar direta ou indiretamente as políticas públicas na área. Sabe-se que um dos desafios atuais é melhorar o acesso para tratamento e consolidar ações preventivas. Ao se estabelecerem políticas públicas baseadas na moralização e que reforcem a estigmatização dos usuários mediante ações muito mais repressivas, em que se confundem e não se diferenciam o tráfico e o uso, pode-se levar a uma exclusão ainda maior e à impossibilidade de se promover a saúde em algum grau dos usuários ${ }^{28}$.

É importante ainda ressaltar que a sociedade (e os profissionais e gestores em algum grau) lida de forma ambígua, em especial, com o uso de álcool. Por um lado, existe uma glamorização e incentivo ao uso de álcool por grupos, sociedade e até, em alguns momentos, pela mídia, criando uma cultura do uso pesado entre a população. Por outro lado, quando esse consumo se torna um problema, esse uso passa ter conotação negativa, associando-se a uma imagem de fraqueza moral e individualização de um problema que é julgado e o usuário é "condenado" à exclusão social.

Concluindo, o estudo e o aprofundamento de aspectos socioculturais envolvidos no uso de álcool e outras drogas são fundamentais para o entendimento de como os usuários são cuidados/excluídos pelos profissionais, como alguns modelos de prevenção e tratamento são implementados e como são definidas e priorizadas algumas políticas públicas. Por fim, o estudo sobre o processo de estereotipização e moralização por parte dos profissionais em relação aos usuários de álcool se torna relevante, pois pode fornecer informações importantes para o estabelecimento de estratégias adequadas de mudança de atitudes desses profissionais e melhoria da qualidade do atendimento aos usuários de álcool.

\section{REFERÊNCIAS}

1. Gigliotti A, Bessa MA. Síndrome de dependência do álcool: critérios diagnósticos. Rev Bras Psiq. 2004;22(1):31-7.

2. Meloni JN, Laranjeira R. Custo social e de saúde do consumo do álcool. Rev Bras Psiq. 2004;26(Supl. 1):7-10

3. Ronzani TM. Avaliação de um processo de implementação de estratégias de prevenção ao uso excessivo de álcool em serviços de atenção primária à saúde: entre o ideal e o possível. [tese] Escola Paulista de Medicina, Universidade Federal de São Paulo, São Paulo; 2005.

4. Babor TF. Linking science to policy. The role of international collaborative research. Alc Res Health. 2002;26(1):66-74.

5. Berger M, Wagner TH, Baker LC. Internet use and stigmatized illness. Soc Sci Med. 2005;61:1821-7.

6. Fortney J, Mukherjee S, Curran G, Fortney S, Han X, Booth BM. Factors associated with perceived stigma for alcohol use and treatment among at-risk drinkers. Behav Health Serv Res. 2005;31(4):418-29. 
7. Room R. Taking account of cultural and societal influences on substance use diagnoses and criteria. Addiction. 2006;101(Suppl 1):31-9.

8. Neves DP. Alcoolismo: acusação ou diagnóstico? Cad Saude Publica. 2004;20(1):7-14.

9. Lima MEO, Vala J. Serão os estereótipos e o preconceito inevitáveis? 0 monstro da automaticidade. In: Lima ME0, Pereira ME (Orgs.). Estereótipos, preconceitos e discriminaçãa. Perspectivas teóricas e metodológicas. Salvador: EDUFBA; 2004, p. 41-68.

10. Williams JR. Effects of labeling the "drug-abuser": an inquiry. Rockville: NIDA; 1976.

11. Amaral MB, Ronzani TM, Formigoni MLOS. The implementation process of screening and brief intervention programs for alcohol abusers in primary health care [resumo]. Alcohol Clin Exp Res. 2007;31(6):117A-117A.

12. Babor T, et al. Alcohol: no ordinary, no commodity. Research and Public Policy. New York: WHO; 2003.

13. Babor T, Higgins-Biddle JC. Intervenções breves: para o uso de risco e nocivo de álcool manual para uso em atenção primáría. Ribeirão Preto: PAI-PAD; 2003.

14. Baker A, Boggs TG, Lewin TJ. Randomized controlled trial of brief cognitive-behavioural interventions among regular users of amphetamine. Addiction. 2001;96:1279-87.

15. Bashir K, King M, Ashworth M. Controlled evaluation of brief intervention by general practitioners to reduce chronic use of benzodiazepines. Br J Gen Pract. 1994;44:408-12.

16. Copeland J, Swift W, Roffman R, Stephens R. A randomized controlled trial of brief cognitive-behavioural interventions for cannabis use disorder. J Subst Abuse Treat. 2001;21(2):55-64

17. Fleming MF, Mundt MP, French MT, Manwell LB, Stauffacher EA, Barry KL. Benefit-cost analysis of brief physician advice with problem drinkers in primary care settings. Med Care. 2000;38(1):7-18

18. Ronzani TM, Ribeiro MS, Amaral MB, Formigoni MLOS. Implantação de rotinas de rastreamento do uso de risco de álcool e de uma intervenção breve na atenção primária à saúde: dificuldades a serem superadas. Cad Saude Publica. 2005;21(3):852-61.

19. Corradi-Webster CM, Minto EC, Aquino FMC, Abade F, Yosetake LL, Gorayeb R, et al. Capacitação de profissionais do programa de saúde da família em estratégias de diagnóstico e intervenções breves para o uso problemático de álcool. SMAD. 2005;1(1):10. Disponível em: http://www2.eerp.usp.br/resmad/artigo_titulo.asp?rmr=49. Acessado em: 29 maio 2007.

20. Minto EC, Corradi-Webster CM, Gorayeb R, Laprega MR, Furtado EF. Intervenções breves para 0 uso abusivo de álcool em atenção primária. Epidemiol Serv Saúde. 2007;16(3):207-20.

21. Bandura A. Modificação do comportamento. Rio de Janeiro: Interamericana; 1979.

22. Rodrigues A, Assmar EML, Jablonski B. Psicologia social. Petrópolis: Vozes; 2002.

23. Marques ACPR, Furtado EF. Intervenções breves para problemas relacionados ao álcool. Rev Bras Psiq. 2004;26(Supl. 1):28-32.

24. Ronzani TM, Furtado EF. A intervenção breve na UBS: quem pode aplicá-la? In: SENAD. Sistema para Detecção do Uso Abusivo e Dependência de Substâncias Psicoativas: encaminhamento, intervenção breve, reinserção social e acompanhamento, Brasília, DF: SENAD 2006, p. 49-57.

25. Room R. Stigma, social inequality and alcohol and drug use. Drug Alcohol Rev 2005;24:143-55.

26. Goffman E. Estigma. Notas sobre a manipulação da identidade deteriorada. 2. ed. Rio de Janeiro: Zahar; 1978

27. Ronzani TM, Andrade T. A estigmatização associada ao uso de substâncias como obstáculo à detecção, prevenção e tratamento. In: SENAD. Sistema para Detecção de Uso Abusivo e Dependência de Substâncias Psicoativas, Braślia, DF: SENAD; 2006, p. 25-32.

28. Dovidio JF, Major B, Crocker J. Stigma: introduction an overview. In: Heatherton TF, Kleck RE, Hebl MR, Hull JG (Orgs.). Social psychology of stigma. New York: Guilford Press; 2003, p. 1-28.
29. Palm J. Moral concerns - Treatment staff and user perspectives on alcohol and drug problems [tese]. Department of Criminology, University of Stockholm, Sthockholm; 2006.

30. Dinos SM, Stevens S, Serfaty M, Weich S, King M. Stigma: the feelings and experiences of 46 people with mental illness. Qualitative study. Br J Psychiatry. 2004;184:176-81.

31. Heatherton TF, Kleck, RE, Hebl MR, Hull JG (orgs). Social psychology of stigma. New York: Guilford Press; 2003.

32. Major B, O'Brien LT. The social psychology of stigma. Annu Rev Psychol. 2005;56: 393-421.

33. Ros M, Gouveia VV (Orgs.). Psicologia social dos valores humanos. São Paulo: Senac; 2006.

34. Ajzen I. Nature and operation of attitudes. Annu Rev Psychol. 2001;52:27-58.

35. Rokeach M. Crenças, atitudes e valores. Teoria de organização e mudança. Rio de Janeiro: Interciência; 1981.

36. Ramos A. Introdução à psicologia social. São Paulo: Casa do Psicólogo; 2003.

37. Thompson JB. Ideologia e cultura moderna. Teoria social crítica dos meios de comunicação de massa. Petrópolis: Vozes; 1995.

38. Hilton JL, Hippel W. Stereotypes. Annu Rev Psychol.1996;47:237-71.

39. Lima ME0, Pereira ME. Estereótipos, preconceitos e discriminação. Perspectivas teóricas e metodológicas. Salvador: EDUFBA; 2004.

40. World Health Organization. Global Status Report on Alcohol. Geneva: World Health Organization; 2001.

41. World Health Organization. Global Status Report on Alcohol. Geneva: World Health Organization; 1999.

42. Carlini EA, Galduróz JC. II levantamento domiciliar sobre 0 uso de drogas psicotrópicas no Brasil: estudo envolvendo as 108 maiores cidades do país. Brasília: Secretaria Nacional Antidrogas (SENAD); 2007

43. Laranjeira $R$, et al. I levantamento nacional sobre os padrões de consumo de álcool na população brasileira. Brasília: SENAD; 2007.

44. Galduróz JCF, et al. V levantamento sobre o consumo de drogas psicotrópicas entre estudantes do ensino fundamental e médio nas 27 capitais brasileiras. Braślia: SENAD; 2005.

45. Ritsher JB, Phelan JC. Internalized stigma predicts erosion of morale among psychiatric outpatients. Psychiatr Res. 2004;129:257-65.

46. Barkin SL, Smith KS, DuRant RH. Social skills and attitudes associated with substance use behaviors among young adolescents. J Adolesc Health. 2002;30:448-54.

47. Magnabosco MB, Formigoni MLOS, Ronzani TM. Avaliação dos padrões de uso de álcool em usuários de serviços de Atenção Primária à Saúde de Juiz de Fora e Rio Pomba (MG). Rev Bras Epidem. 2007;10(4):637-47.

48. Bendtsen P, Akerlind I. Changes in attitudes and practices in primary health care with regard to early intervention for problem drinkers. Alcohol Alcohol. 1999;34(5):795-800.

49. Link BG, et al. The consequences of stigma for the self-esteem of people with mental illnesses. Psychiatr Serv. 2001;52(12):1621-6.

50. Barney LJ, Griffiths KM, Jorm AF, Christensen H. Stigma about depression and its impact on help-seeking intentions. Aust N Z J Psychiatry. 2006;40:51-4.

51. Johnstone M. Stigma, social justice and the rights of the mentally ill: challenging the status quo. Aust N Z J Ment Health Nurs. 2001;10:200-9.

52. Corrigan PW, Lurie BD, Goldman HH, Slopen N, Medasani K, Phelan S. How adolescents perceive the stigma of mental illness and al cohol abuse. Psychiatr Serv. 2005;56(5):544-50.

53. Aalto M, Pekuri P, Seppa, K. Primary health care professionals' activity in intervening in patients' alcohol drinking during a 3-year brief intervention implementation project. Drug Alcohol Depend. 2003;69(1):9-14 